\title{
Combining Genetic Profiles and Energy Expenditure Measurements to Guide Weight Loss Management Programs
}

\begin{abstract}
Robert A. Orlando
Department of Biochemistry and Molecular Biology University of New Mexico, School of Medicine, MSC08 4670, University of New Mexico, Albuquerque, New Mexico, 87131, USA

*Corresponding author: Orlando RA, Department of Biochemistry and Molecular Biology University of New Mexico, School of Medicine, MSC08 4670, University of New Mexico, Albuquerque, New Mexico, 87131, USA, Tel: 505-272-5593; Fax: 505-272-5593; E-mail: rorlando@salud.unm.edu

Received date: Jul 28, 2014; Accepted date: Jul 29, 2014; Published date: Jul 29, 2014

Copyright: (c) 2014 Orlando RA. This is an open-access editorial distributed under the terms of the Creative Commons Attribution License, which permits unrestricted
\end{abstract} use, distribution, and reproduction in any medium, provided the original author and source are credited.

\section{Editorial}

In our present era of widespread obesity, it is without doubt we are all well acquainted with dietary struggles faced by our patients, friends, and family members as they follow the latest commercialized trend trying earnestly to shed those extra pounds. To state the obvious, dieting to achieve permanent weight loss is hard, requiring diligence, a sound plan, and proper support to succeed. But is this all it takes? We hear many stories expressing long-term commitment to well-planned diets that have been assembled by trained nutritionists and dieticians. And of course, encouragement is readily offered by family and friends. Yet, in spite of these well intentioned pursuits, dieting largely fails, often with the added consequence of rebounding to an even greater weight than pre-diet measures. This leads to expressions of anger, frustration, resignation, and confusion. Besides the physical toll, the effect on emotional well-being is inevitable and very often poorly addressed. Perhaps we need to look at this problem from a different perspective; as Albert Einstein taught us, we cannot solve our problems with the same thinking that created them.

Weight management designs follow a relatively simple equation. Energy, quantified by caloric content, is consumed and these ingested calories are used as fuel to perform bodily functions. When energy consumed outpaces energy used, then the excess is stored for later needs and weight gain occurs. Conversely, when energy expenditure exceeds energy consumed, then stored energy is mobilized to make up the difference and weight loss results. Most people know this; however, when a dieter consumes fewer calories, why do they often struggle to lose weight? They rightfully claim, "I'm eating less but not losing weight. It doesn't make sense according to the science I learned". Perhaps these individuals don't quite have all the information necessary for success and would benefit with more information that current science can provide.

The age-old adage claiming "we are what we eat" has become much more complex when considering the unprecedented detail provided by genomics combined with more sensitive methods in determining an individual's metabolic activity. Energy consumed still must equal energy expended to maintain weight; this is a fundamental physiologic principle. However, we now have more advanced knowledge showing that every individual possesses genetic modifications, mostly in the form of nucleotide polymorphisms that can impart unique properties to the functionality of their gene products. Some of these nucleotide changes can result in gain-of-function or loss-of-function to particular enzymes, or ever-so-slight modifications to their catalytic activities that can make large differences over decades. In addition, variations in nucleotide sequence within promoter elements can disrupt transcriptional regulation of metabolic proteins and alter overall expression levels. Now imagine if these polymorphisms dictate an individual's metabolic activities so that each of us is unique as to how much energy we can store and how much we can utilize. Without this individualized knowledge, it would seem only best-guess to predict a response to even the most professionally constructed weight management plan.

Genome-Wide Association Studies (GWAS) began in 2005 and tested the associations of millions of common variants with obesity and other obesity-related traits by comparing the frequencies of these variants in obese cases with normal weight controls. To date, GWAS has identified $>30$ genetic loci that demonstrate strong positive association for Body-Mass Index (BMI) measurements [1,2]. Whether these variants are causal in creating a positive energy imbalance resulting in weight gain or are passively associated with obesity remains to be determined; although the latter is more likely since the effect sizes of these loci are much smaller than originally anticipated (typically $<5 \%$ ). Thus, the original hypothesis that mutations in single genes might be identified that predispose an individual to obesity, hence the monogenic hypothesis, is likely only to be true in very rare forms of obesity. Rather, the identification of numerous genetic variants with small effect sizes suggests that the most common forms of obesity are polygenic in nature. Adding to this complexity are the more recent discoveries of epigenetic changes that modify metabolic gene expression $[3,4]$. Unlike the heritability constraints of genetic changes, epigenetic modifications can occur within an individual's lifetime and are often determined by dietary and environmental exposures [5]. Together, these collective changes create a unique blueprint that defines each individual's metabolic activities. Because of this individualized blueprint, we can envisage that each of us will respond differently to caloric intake, providing a hypothesis as to why some of us respond quickly and efficiently to dietary modifications, while others struggle with weight gain even when drastically curtailing calorie consumption.

It is reasonable to posit that the genetic determinants that dictate our basal metabolic rates, thermogenic activities, and fuel expenditures at rest and during physical activity represent vital scientific information that would provide those desiring to lose weight with a guide to personalize their food consumption and energy expenditure to best adapt to their unique metabolic profile. With knowledge of these genetic determinants, we can expand our original energy equation, i.e. weight management is simply dependent on balancing calorie consumption with energy expenditure, by adding an individual's genetic blueprint to this equation and in doing so personalize their weight management program to accommodate their personal genetic profile. Translation of this information into the Clinical setting will be a challenge, yet not insurmountable. The first 
requirement will be to obtain a complete genetic profile for each patient, or at the very least, a profile of the known obesity genes for comparison with identified polymorphisms. Although ethical considerations continue to be debated, accessibility of whole genome sequences for individuals is becoming increasingly affordable and within reasonable time frames [6-8]. However, we must keep in mind that genetic information will only provide limited knowledge; primarily a risk analysis for developing obesity, and with additional studies, maybe predictive value for successful weight loss.

For a more complete picture of an individual's metabolic landscape to decide on the best course of action for weight loss, additional metabolic measurements will be necessary, such as metabolic rates while at rest and during activity. These metabolic rates, often termed energy expenditure, define the net amount of energy an individual uses to maintain normal biological functions [9,10]. An accurate assessment of energy expenditure would provide a better understanding of caloric needs and inform a better design for dietary requirements to optimize the weight loss plan [11]. Energy expenditure is the sum of three main components including basal metabolic rate, diet-induced thermogenesis, and physical activity. Measuring energy expenditure can be achieved with two methods: calorimetry, by measuring heat loss; or indirect calorimetry, by measuring gas exchanges during respiration. Calorimetry requires tightly-controlled, environmental chambers that are currently impractical for clinical applications. However, indirect calorimetry has been successfully used for quantifying energy expenditure for clinical applications and research studies in field settings $[12,13]$. The basic premise of indirect calorimetry relies on the fact that metabolic conversion of food energy has a defined heat of combustion per quantity of oxygen used, and the value of this measurement is dependent on which substrate/fuel is being oxidized, whether it be carbohydrate, protein or fat. By measuring oxygen consumption (VO2) and carbon dioxide excretion (VCO2), a Respiratory Quotient (RQ) is calculated by determining $\mathrm{VCO} 2$ / VO2, and the net energy released from the fuels can be expressed as calories (or kilocalories, kcal or KJ) per unit of time [14]. Clinical measurements of resting energy expenditure are performed using mobile indirect calorimetry systems that quantify both oxygen and carbon dioxide volumes [15]. The most significant challenge to measuring energy expenditure in a clinical setting is the individual's ability to remain calm and breathe normally throughout the measurement period, which typically lasts for 20-30 min.

The information provided by genomic studies and the effects of gene variants on metabolic activities has made us more acutely aware of how subtle changes can have substantial impact on clinical presentations. The impact of these variants is often greatest in metabolic diseases such as obesity, and obesity-dependent diseases such as Type II Diabetes Mellitus and atherosclerotic cardiovascular disease. These diseases can take decades of chronic metabolic imbalance to fully manifest, and because of this, supports a need for a complete and thorough analysis of an individual's genetic predisposition and periodic measurement of metabolic activity. Genetic analysis only needs to be done once in an individual's lifetime, and metabolic rate analysis possibly every ten years, adjusted for co-morbidities and aging. Periodic measurements of energy expenditure are necessary because resting levels are known to decrease by approximately $2-4 \%$ with each decade of life $[16,17]$. In addition, since body composition, thyroid status, disease states and drug treatment regimens can impact energy expenditure, an individual's health status will also need consideration for useful assessment.
The obesity epidemic has maintained a relentless grip on our population and presents challenges for reversal that are well beyond the average person's ability to meet. Right now individuals pursuing weight loss are generally offered one-size-fits-all dietary suggestions, generic exercise plans, and plenty of encouraging words. With current obesity trends, this path is only having marginal effects [18]. The best efforts for consumers to shun those foods with high caloric content likely take place when they have greater knowledge about their personal genetic makeup and metabolic phenotype. For example, the presence of obesity-associated nucleotide variants in one of the major obesity genes (FTO) were examined in a cohort of overweight women (BMI>25) [19]. Those with high-risk variants expressed relief knowing they were susceptible to weight gain because of their genetic predisposition; however, with this information they also expressed increased motivation to overcome challenges imposed by their genetic makeup. Perhaps empowering our overweight and obese patients, friends, and family members with combined knowledge of their personal metabolic phenotype and genetic disposition will provide them with the confidence and support needed to embrace the challenges of permanent weight loss.

\section{Acknowledgements}

The author declares no competing financial interests.

\section{References}

1. Waalen J (2014) The genetics of human obesity. Transl Res DOI: 10.1016/ j.trsl.2014.05.010.

2. Xia Q, Grant SF (2013) The genetics of human obesity. Ann N Y Acad Sci 1281: 178-90.

3. Drummond EM, Gibney ER (2013) Epigenetic regulation in obesity. Curr Opin Clin Nutr Metab Care 16: 392-7.

4. Schwenk RW, Vogel H, Schurmann A (2013) Genetic and epigenetic control of metabolic health. Mol Metab 2: 337-47.

5. Youngson NA, Morris MJ (2013) What obesity research tells us about epigenetic mechanisms. Philos Trans R Soc Lond B Biol Sci 368 DOI: 10.1098/rstb.2011.0337.

6. Joly Y, Saulnier KM, Osien G, Knoppers BM (2014) The ethical framing of personalized medicine. Curr Opin Allergy Clin Immunol DOI: 10.1097/aci. 0000000000000091.

7. Prince AE, Roche MI (2014) Genetic Information, Non-Discrimination, and Privacy Protections in Genetic Counseling Practice. J Genet Couns DOI: 10.1007/s10897-014-9743-2.

8. Johansen Taber KA, Dickinson BD, Wilson M (2014) The promise and challenges of next-generation genome sequencing for clinical care. JAMA Intern Med 174: 275-80.

9. Psota T, Chen KY (2013) Measuring energy expenditure in clinical populations: rewards and challenges. Eur J Clin Nutr 67: 436-42.

10. Kinney J, Tucker H, eds. Energy Metabolism: Tissue Determinants and Cellular Corollaries. 1991, Raven Press: New York, NY.

11. Laddu D, Dow C, Hingle M, Thomson C, Going S (2011) A review of evidence-based strategies to treat obesity in adults. Nutr Clin Pract 26: $512-25$.

12. Brychta R, Wohlers E, Moon J, Chen K (2010) Energy expenditure: measurement of human metabolism. IEEE Eng Med Biol Mag 29: 42-7.

13. Leonard WR (2012) Laboratory and field methods for measuring human energy expenditure. Am J Hum Biol 24: 372-84.

14. Weir JB (1949) New methods for calculating metabolic rate with special reference to protein metabolism. J Physiol 109: 1-9.

15. Van Remoortel H, Giavedoni S, Raste Y, Burtin C, Louvaris Z, et al. (2012) Validity of activity monitors in health and chronic disease: a systematic review. Int J Behav Nutr Phys Act 9: 84-97. 
Citation: Orlando RA (2014) Combining Genetic Profiles and Energy Expenditure Measurements to Guide Weight Loss Management Programs. J Biomol Res Ther 3: e135. doi:10.4172/2167-7956.1000e135

Page 3 of 3

16. Tzankoff SP, Norris AH (1978) Longitudinal changes in basal metabolism in man. J Appl Physiol Respir Environ Exerc Physiol 45: 536-9.

17. Roberts SB, Dallal GE (2005) Energy requirements and aging. Public Health Nutr 8: 1028-36.

18. Morgen CS, Sorensen TI (2014) Obesity: Global trends in the prevalence of overweight and obesity. Nat Rev Endocrinol DOI: 10.1038/nrendo. 2014.124.
19. Meisel SF, Wardle J (2014) Responses to FTO genetic test feedback for obesity in a sample of overweight adults: a qualitative analysis. Genes Nutr 9: 374 . 